\title{
FORMATION OF HIERARCHICAL CUO NANOWIRES ON A COPPER SURFACE VIA A ROOM-TEMPERATURE SOLUTION-IMMERSION PROCESS
}

\author{
S. FUENTES $S^{a *}$, R. A. ZÁRATE ${ }^{a}$, P. MUÑOZ ${ }^{b}$ AND D. E. DÍAZ-DROGUETT \\ a Departamento de Física, Facultad de Ciencias, Universidad Católica del Norte, Casilla 1280, Antofagasta, Chile.

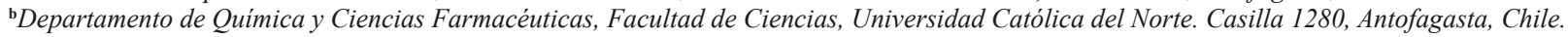 \\ 'Departamento de Física, Facultad de Ciencias Físicas y Matemáticas, Universidad de Chile. Casilla 487-3, Santiago, Chile.
}

(Received: September 14, 2009 - Accepted: December 30, 2009)

\begin{abstract}
$\mathrm{CuO}$ nanowires were successfully made through a simple wet chemical method at room temperature by immersing on copper sheets in a $4 \mathrm{M}$ ammonia solution for 4 days and then subjecting it to heat treatment. Immersion time and heat treatment have an important effect on the length, diameter, and density of the $\mathrm{CuO}$ nanostructures. X-ray powder diffraction (XRD) patterns indicated that the samples are composed of a single phase, CuO. Scanning electron microscopy (SEM), transmission electron microscopy (TEM), and Raman spectroscopy studies showed that the wet-treated samples consisted of nanofiber-like structures of monoclinic $\mathrm{CuO}$, while the heat-treated samples consisted of well-defined nanowires which also exhibited the monoclinic phase.
\end{abstract}

Keywords: A. Oxides; A. Semiconductors; B. Chemical synthesis.

\section{INTRODUCTION}

One-dimensional (1D) nanomaterials, such as nanotubes, nanowires, and nanobelts or nanoribbons have received much attention because they are considered to be the key structural components of future electronic, optical, and mechanical devices ${ }^{1}$. Their unique properties can be harnessed for the design and fabrication of nanosensors, solar cells, field emitters, and transistors ${ }^{2-4}$.

Among the materials based on $3 \mathrm{~d}$ transition metals, cupric oxide $(\mathrm{CuO})$ is of great interest due to its catalytic properties and also because of its close connection with high-temperature superconductors ${ }^{5,6} . \mathrm{CuO}$ is a p-type semiconductor with a band gap of $1.2 \mathrm{eV}$, and since this gap is smaller than $1.85 \mathrm{eV}$, this compound is being studied for its field emission properties? On the other hand, the large surface area, excellent stability, low production cost, and good electric properties of nanostructured $\mathrm{CuO}$ have fueled new studies to determine its applicability as material for solar cells, in particular due to its photoconductivity and photochemical properties ${ }^{8-10}$. Based on these applications, many methods have been developed to prepare $\mathrm{CuO}$ with various morphologies ${ }^{11-15}$.

Recent research indicates that 2D and 3D structures of $\mathrm{CuO}$ nanomaterials have been extensively studied. In particular, self-assembled complex $\mathrm{CuO}$ architectures, such as 3D peanut-like patterns ${ }^{16}$, picky microspheres ${ }^{17}$, nanodendrites ${ }^{18}$, chrysanthemum-like architectures ${ }^{19}$, dandelion-shaped ${ }^{20}$, hollow structures ${ }^{21}$, have attracted considerable attention due to their fundamental importance and potential future applications.

Very recently, few reports demonstrated that $\mathrm{CuO}$ architectures with higher dimensionality and complexity could be synthesized on $\mathrm{Cu}$ surfaces in a controlled fashion ${ }^{22}$. However, the obtained morphology (ellipsoid, flower, dendrite) in these reports was limited and the preparation process usually involved surfactants or additives. The realization of novel morphology such as those observed in natural materials or biominerals on $\mathrm{Cu}$ foils by a surfactantsfree route will be of fundamental and practical importance ${ }^{23}$.

On the other hand, direct and simple thermal oxidation methods have been used to synthesize $\mathrm{Cu}(\mathrm{OH})_{2}$ and $\mathrm{CuO}$ nanofibers and nanorods. Using this convenient route, with no catalyst and template assisted, many research teams successfully prepared $\mathrm{CuO}$ nanowire by oxidizing copper plates under different conditions such as different annealing temperatures, times, or atmospheres ${ }^{24-28}$.

In this paper we report the synthesis of well- defined $\mathrm{CuO}$ nanowire on copper sheets using a simple wet chemical method at room temperature, and the effect of heat treatment on the final surface morphology. The morphology and dimensionality of $\mathrm{CuO}$ nanoarchitecture can be tuned by simple variation of reaction parameters.

\section{EXPERIMENTAL SECTION}

In a typical experiment, duplicate $1 \mathrm{~cm} \times 1 \mathrm{~cm}$ copper sheets (TCL., purity: $99.98 \%$, thickness: $0.4 \mathrm{~mm}$ ) were washed with $1 \mathrm{M} \mathrm{HCl}$ solution for 15 min and subsequently with deionized water to remove surface impurities. The washed copper foils were then immersed in $20 \mathrm{~mL}$ of ammonia solution $4 \mathrm{M}$ for different times (from 2 to 5 days) at room temperature.

After the reaction, the copper sheets were removed from the solution, rinsed with deionized water, and air dried. A sample of each sheet was heated at $120{ }^{\circ} \mathrm{C}$ for $1 \mathrm{~h}$ and then at $180{ }^{\circ} \mathrm{C}$ for $1 \mathrm{~h}$. After the furnace had cooled to room temperature, the copper sheets had a black film on the surface.

The as-prepared samples were characterized by X-ray powder diffraction (XRD on a Siemens D5000 diffractometer with CuK radiation $(40 \mathrm{kV}, 30$ $\mathrm{mA}$ ). The morphology of the samples was examined using a Low Vacuum Scanning Electron Microscopy (LV-SEM, JSM-6360LV) equipped with an EDX detector. Transmission electron microscopy (TEM) studies were made on a Tencai F20 FEG-TEM operated at $200 \mathrm{kV}$ equipped with an EDS detector. The TEM samples were prepared by suspending the powder in ethanol and then collecting it on a carbon grid. The samples were ultrasonically dispersed in isopropanol for TEM. Raman Spectra were recorded on a WITEC model CRC200 using a $5.5 \mathrm{~mW}$ laser with a wavelength of $514.5 \mathrm{~nm}$.

\section{RESULTS AND DISCUSSION.}

The surface morphology of the $\mathrm{CuO}$ on the copper sheet after immersion in the ammonia solution and subsequent heat treatment were investigated by Scanning Electron Microscopy (SEM), as shown in Fig. 1. The dependence of surface morphology on immersion time and was studied to gain a clear view of the nanofiber formation process.

As shown in Fig. 1a, there was no presence of any nanostructure on the copper sheets by day 2 of treatment. Only a few nanofibers were observed by day 3 (Fig. 1b). As immersion time increased, by day 4 the surface coverage of nanofibers became denser (Fig. 1c). However, when growth time was extended to day $5, \mathrm{CuO}$ nanofiber growth became much slower and more compacted, as shown in Fig. 1d. From these results we concluded that immersion time has an important influence on the length, diameter, and density of the nanofibers.
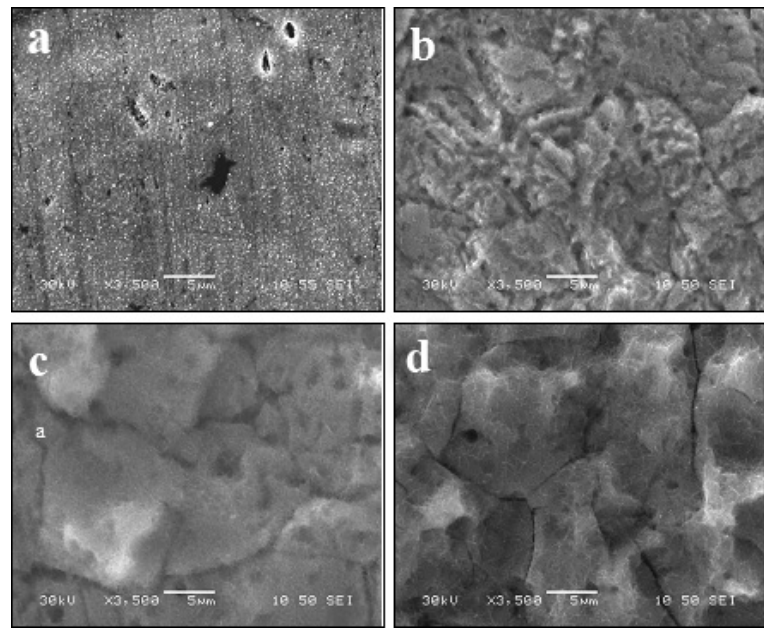

Figure 1. The surface morphology evolution of the $\mathrm{CuO}$ at different duration times (days): (a) $2 \mathrm{~d}$, (b) $3 \mathrm{~d}$, (c) $4 \mathrm{~d}$ and d) $5 \mathrm{~d}$, respectively. 
To investigate the effect of heat treatment on the final surface morphology of the copper samples, SEM images of a copper sheet after heat treatment at $200{ }^{\circ} \mathrm{C}$ are shown in Fig. 2. By day 4, Fig. 2a, the treated sample shows that the $\mathrm{CuO}$ nanofiber array has a width of $90 \mathrm{~nm}$ to $100 \mathrm{~nm}$ and a length of several micrometers. Fig. $2 \mathrm{~b}$ shows that the heat treated sample has undergone slight modifications on the surface, with the width of nanofibers decreasing to $\sim 60$ $\mathrm{nm}$.
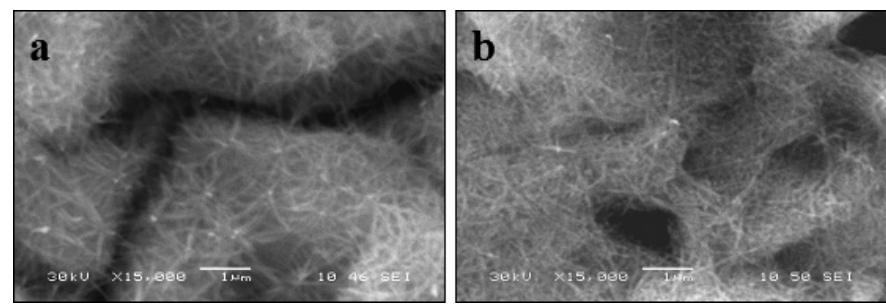

Figure 2. SEM image of $\mathrm{CuO}$ nanostructures grown on copper sheets treated at 4 days. (a) without and (b) with heat treatment.

Typical XRD patterns of the synthesized $\mathrm{CuO}$ samples are shown in Fig. 3. Curves $3 \mathrm{a}$ and $3 \mathrm{~b}$ correspond to samples treated at 4 days (4D) without and with heat treatment, respectively. All the XRD peaks shown can be indexed as monoclinic $\mathrm{CuO}$ (space group $\mathrm{C} 2 / \mathrm{c}$; JCPDS cards no. 48-1548). The XRD pattern of the heat treated sample has sharper peaks than the sample without heat treatment. Peak broadening in diffraction lines of the sample under heat treatment may be related to a size reduction of $\mathrm{CuO}$ crystals. According to the standard $\mathrm{XRD}$ pattern of the $\mathrm{CuO}$ powder, the peaks at $35.5^{\circ}, 38.7^{\circ}, 48.8^{\circ}$ and $58.4^{\circ}$ can be attributed to the Miller indexes of $(-111),(200),(-202)$ and (202). Notice that the peaks at $2 q$ values $\left(43.3^{\circ}\right.$ and $\left.50.4^{\circ}\right)$ are due to the copper substrate (marked with an asterisk). No reflections from impurities $\left(\mathrm{Cu}(\mathrm{OH})_{2}\right.$ or $\mathrm{Cu}_{2} \mathrm{O}$ ) were detected in the samples.

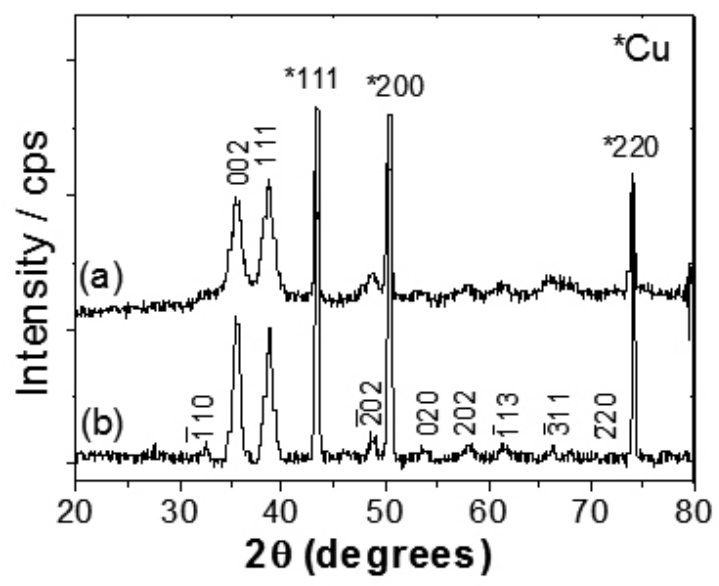

Figure 3. XRD pattern of $\mathrm{CuO}$ nanostructures grown on copper sheets treated at 4 days. (a) without and (b) with heat treatment.

Fig. 4 presents the Raman spectra of the $\mathrm{CuO}$ powder prepared on the copper sheets after immersion and subsequent heat treatment.

$\mathrm{CuO}$ belongs to the $\mathrm{C}_{2 \mathrm{~h}}{ }^{6}$ space group with two molecules per primitive cell. One can find for the center zone normal modes $\Gamma_{\mathrm{Pu}}=4 \mathrm{Au}+5 \mathrm{Bu}+\mathrm{Ag}+2 \mathrm{Bg}$. There are three acoustic modes $(\mathrm{Au}+2 \mathrm{Bu})$, six infrared actives modes $(3 \mathrm{Au}+3 \mathrm{Bu})$, and three Raman active modes $(\mathrm{Ag}+2 \mathrm{Bg})$.

It is seen that there are three Raman active optical phonons at 278,321 and $608 \mathrm{~cm}^{-1}$ in Fig. 4a. We can assign the peak at $278 \mathrm{~cm}^{-1}$ to the Ag mode and the peaks at 321 and $608 \mathrm{~cm}^{-1}$ to the $\mathrm{Bg}$ modes. The Raman peak positions of $\mathrm{CuO}$ are dependent on the method of preparation [29-30]. When the $\mathrm{CuO}$ powder was thermally treated, the Raman peak became stronger and sharper and shifted slightly to higher frequencies: 287,332 and $625 \mathrm{~cm}^{-1}$ (Fig. 4b).

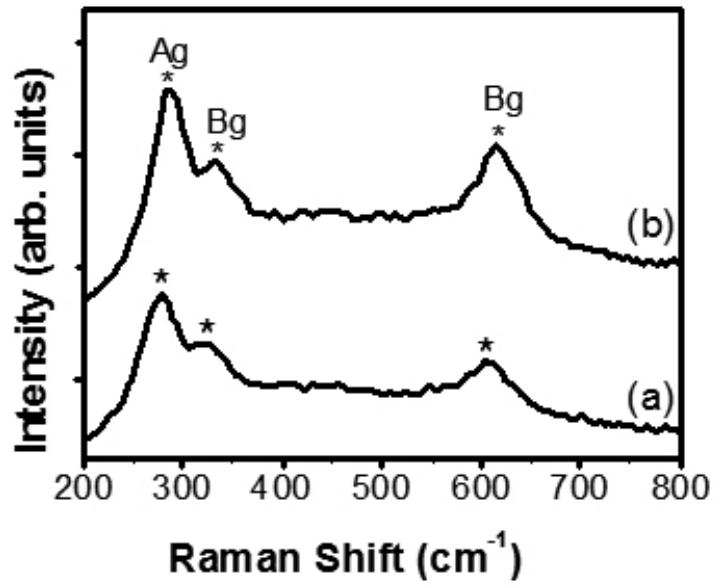

Figure 4. Raman spectra of $\mathrm{CuO}$ nanostructures grown on copper sheets treated at 4 days. (a) without and (b) with heat treatment.

Fig. 5 shows TEM images of a sample treated at $4 \mathrm{D}$ without heat treatment. Fig. 5a is a bright field image showing an agglomerated of nanoparticles together with rod-like structures, which lies on the zone indicated by the arrow. TEM Images at higher magnification (not shown) revealed particles sizes around $7 \mathrm{~nm}$ with shapes not faceted. Fig. $5 \mathrm{~b}$ is a selected area electron diffraction (SAED) pattern coming from Fig. 5a. This SAED pattern shows the formation of diffraction rings, whose weak intensities reveal the low cristallinity of the sample. The indexed diffraction rings can be attributed to (002), (200) and $(-113)$ planes of monoclinic $\mathrm{CuO}$, corroborating the XRD and Raman results.

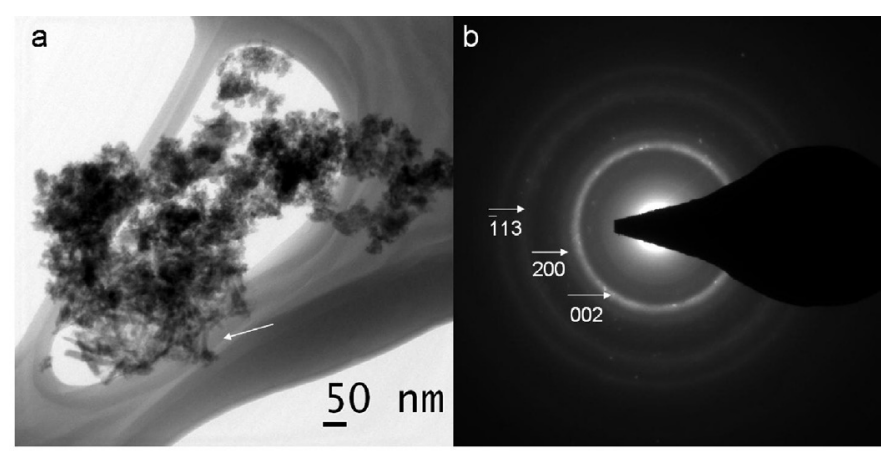

Figure 5. TEM images of a sample treated at 4 days without heat treatment. (a) Bright field image and (b) SAED pattern.

Fig. 6 shows TEM images of a sample treated at $4 \mathrm{D}$ with heat treatment. Fig. $6 \mathrm{a}$ is a bright field image showing interlinked nanowires mixture with some agglomerated particles with widths that not exceeds the $10 \mathrm{~nm}$. Fig. $6 \mathrm{~b}$ is the SAED pattern corresponding to bright field of Fig. 6a. This pattern depicts more defined diffraction rings, and its stronger intensities compared with the rings of the SAED pattern of Fig. 5b, is due to the higher cristallinity of the sample that has been thermally treated. In this case, the indexed diffraction rings can be again attributed to $(-111),(-202),(202)$ and $(022)$ planes of monoclinic $\mathrm{CuO}$. Fig. 6c is a high resolution TEM (HRTEM) image, of the framed part in Fig. 6a, which displays lattice fringes with different crystallographic orientations and corroborate that the formation process of the wires-like structures is produced by a coalescence phenomenon stimulated by the heat treatment undergoes for this sample. The HRTEM image shows, moreover, a zone whose lattice fringes displays an interplanar spacing of $2.51 \AA$, this value match the interplanar distance $(-111)$ of monoclinic $\mathrm{CuO}$. 


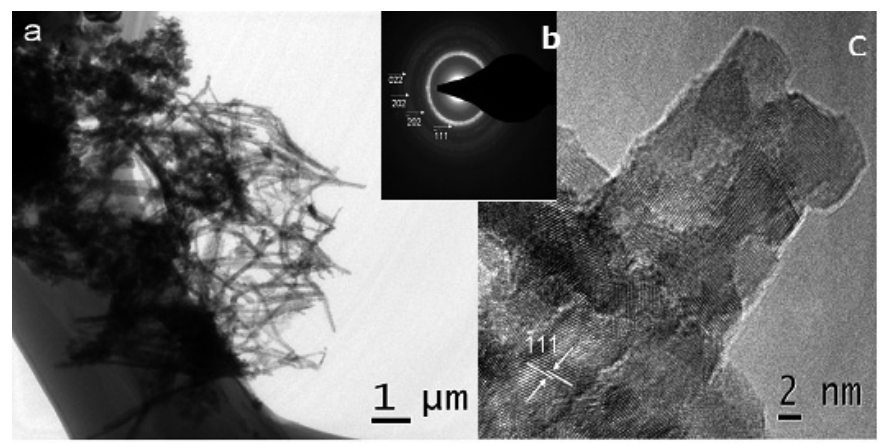

Figure 6. TEM images of the sample treated at 4 days with heat treatment. (a) Bright field image, (b) SAED pattern of the wire shown in (a) and (c) HRTEM image.

The formation mechanism of $\mathrm{CuO}$ involves a simple process which can be illustrated as follows:

$$
\begin{aligned}
& \mathrm{Cu}+\mathrm{O}_{2}+\mathrm{H}_{2} \mathrm{O}+\mathrm{NH}_{3} \rightarrow\left[\mathrm{Cu}\left(\mathrm{NH}_{3}\right)_{4}\right]^{2+} \\
& \mathrm{Cu}\left[\left(\mathrm{NH}_{3}\right)_{4}\right]^{2+}+2 \mathrm{OH}^{-} \rightarrow \mathrm{Cu}(\mathrm{OH})_{2} \\
& \mathrm{Cu}(\mathrm{OH})_{2} \rightarrow \mathrm{CuO}+\mathrm{H}_{2} \mathrm{O}
\end{aligned}
$$

Reactions (1) and (2) were used previously for the synthesis of $\mathrm{Cu}(\mathrm{OH})_{2}$. In this experiment, the solution used is much more basic, and therefore $\mathrm{Cu}(\mathrm{OH})_{2}$ turns unstable and immediately decomposes into $\mathrm{CuO}$. This process allows excellent adhesion of the $\mathrm{CuO}$ nanostructures to the $\mathrm{Cu}$ substrates.

\section{CONCLUSION}

In summary, we have successfully synthesized $\mathrm{CuO}$ nanowire in situ on copper substrates through a simple solution-immersion step and a subsequent heat treatment. It can be concluded that the effect of the heat treatment on the $\mathrm{CuO}$ has an important influence on the diameter and density of the nanowires, but no obvious effect on their length. The advantages of our methods include low temperature, uniform size, superior adherence, and homogeneous coverage.

\section{ACKNOWLEDGMENTS}

This work was supported by the DGIP of the Universidad Católica del Norte. We acknowledge a grant from Minera Escondida Limitada (MEL 22) and project ESO-AUI. R.A.Z. acknowledges grants from FUNDACIÓN ANDES under contract C-13876.

\section{REFERENCES}

1. Z.W. Pan, Z.R. Dai, Z.L. Wang, Science, 291, 1947 (2001).

2. A. Chowdhuri, P. Sharma, V. gupta, K. Screenivas, K. Rao, J. Appl. Phys. 92, 2172 (2002).

3. S. Bennici, A. Gervasini, Appl. Catal. B. 62, 336 (2006).

4. A. Anandan, X. Wen, S. Yang, Mater Chem. Phys. 93, 35 (2005).

5. H. Yamada, X. Zheng, Y. Soejima, M. Kawaminami, Phys. Rev. B. 69, 104104 (2004).

6. X. Zheng, Y. Kodama, K. Saito, E. Tanaka, Y. Tomokiyo, H. Yamada, C. Xu, Phys. Rev. B. 69, 94510 (2004).

7. C. Hsieeh, J. Chen, H. Lin, H. Shih, Appl. Phys. Lett. 83, 3383 (2003).

8. H. Wang, Q. Pan, J. Zhao, G. Yin, P. Zuo, J. Power Sourc. 167, 206 (2007).

9. J. Switzer, J. Kothari, P. Poizot, S. Nakanishi, E. Bohannan, Nature. 425, 490 (2003).

10. E. Vigil, B. González, I. Zumeta, C. Domingo, X. Doménech, J. Ayllón, Thin Solid Films. 489, 50 (2005).

11. W. Ghim, A. Wong, A. Wee, M. Welland, Nano Lett. 4, 2023 (2004).

12. X. Wang, G. Xi, S. Xiong, Y. Liu, B. Xi, W. Yu, Y. Qian, Cryst. Growth Des. 7, 930 (2007).

13. X. Wu, G. Shi, J. Phys. Chem. B. 110, 11247 (2006).

14. D. Zhang, C. Chen, J. Zhang, F. Ren, Chem. Mater. 17, 5242 (2005).

15. X. Wu, H. Bai, J. Zhang, F. Chen, G. Shi, J. Phys. Chem. B. 109, 22836 (2005).

16. L.Z. Zhang, J.C. Yu, A.W. Xu, Q. Li, K.W. Kwong, S.H. Yu, J. Cryst. Growth. 266, 545 (2004).

17. Y. Xu, D. Chen and X. Jiao, J. Phys. Chem. B. 109, 13561 (2005).

18. S.Z. Li, H. Zhang, Y.J. Ji, D.R. Yang, Nanotechnology. 15, 1428 (2004).

19. Y. Liu, Y. Chu, M.Y. Li, L.H. Dong, J. Mater. Chem. 16, 192 (2006).

20. S. Wang, J. Zhang, C. Chen, Scripta Materialia. 57, 337 (2007).

21. B. Liu, H.C. Zeng, J. Am. Chem. Soc. 126, 8124 (2004).

22. Z.P. Zhang, H.P. Sun, X.Q. Shao, D.F. Li, H.D. Yu, M.Y. Han, Adv. Mater 17, 42 (2005).

23. Z.P. Zhang, X.Q. Shao, H.D. Yu, Y.B. Wang, M.Y. Han, Chem. Mater. 17, 332 (2005).

24. J. Chen, F. Zhang, J. Wang, G. Zhang, B. Miao, X. Fan, D. Yan, P. Yan, J. Alloy Comp. 454, 268 (2008).

25. W. Zhang, S. Ding, Z. Yang, A. Liu, Y. Qian, S. Tang, S. Yang. J Cryst. Growth. 291, 479 (2006).

26. M. Kaur, K. Muthe, S. Despande, S. Choudhury, J. Singh, N. Verma, S. Gupta, J. Yakhmi, J. Cryst. Growth. 289, 670 (2006).

27. W.X. Zhang, X.G. Wen, S.H. Yang, Y. Berta, Z.L. Wang, Adv. Mater. 15, $822(2003)$.

28. Z.R. Tian, J.A. Voigt, J. Liu, B. Mckenzie, M.J. Mcdermott, J. Am. Chem. Soc. 124, 12954 (2002).

29. T. Yu, X. Zhao, Z. Shen, Y. Wu, W. Su, J. Cryst. Growth. 268, 590 (2004).

30. J. Xu, W. Ji, Z. Shen, J. Tang, A. Ye, D. Jia, J. Solid State Chem. 147, 516 (1999). 